\title{
Enterovirus myocarditis as a cause of neonatal collapse
}

\author{
D Inwald, O Franklin, D Cubitt, M Peters, A Goldman, M Burch
}

Arch Dis Child Fetal Neonatal Ed 2004;89:F461-F462. doi: 10.1136/adc.2003.034439

Seven neonates required intensive care at our institution with enterovirus myocarditis, 2001-2003. Presentation was at a median age of 9 days. All had ischaemic electrocardiograms, poor ventricular function, raised creatine kinase, and enterovirus RNA detected by reverse transcriptase polymerase chain reaction. Four survived. Enterovirus myocarditis may be an under recognised cause of neonatal collapse.

$\mathrm{E}$ nteroviruses are single stranded RNA viruses of the picornavirus family. Although most enterovirus infection is subclinical or mild, in the neonatal period, enterovirus can cause meningoencephalitis, sepsis syndrome, hepatitis, or myocarditis. ${ }^{1}$ However, enterovirus myocarditis is not often in the differential diagnosis of neonatal collapse. Here, we report a series of cases of neonatal enterovirus myocarditis presenting to our institution between 2001and 2003. We describe its presentation, management, and pathophysiology.

Seven cases were identified (table 1). Median gestational age was 38 weeks (range 36-40). Five infants were born by spontaneous vaginal delivery, and two by caesarean section. The median age at presentation was 9 days (range 5-18). In six cases there was cardiorespiratory collapse requiring intubation, ventilation, volume resuscitation, and inotropes. In case 7 , the infant presented at 15 days with heart failure and required nasal continuous positive airways pressure and diuretics but no inotropes. Creatine kinase was assayed in five patients, and myoglobin (MB) fraction in three patients. Median creatine kinase was 427 IU/l (222-1344, normal $<120$ ) with a MB fraction of $13 \%(11-25$, normal < 5 ).

All had ischaemic electrocardiograms (fig 1) and on echocardiography had anatomically normal hearts with poorly contractile, dilated left ventricles. Anomalous origin of the coronary arteries was excluded, and coronary flow was normal in all cases. Four patients had paradoxical septal motion, and thus a shortening fraction could not be calculated. The median fractional shortening in the remaining three infants was $10 \%$ with a range of $9-10 \%$ (normal $>30 \%)$.

Four infants had global myocardial dysfunction with a closed arterial duct. Three had a relatively spared right ventricle and a patent arterial duct with right to left flow. The latter infants had single ventricle physiology and were managed with prostaglandin El to maintain ductal patency and avoidance of both hyperoxia and alkalosis to maintain the systemic circulation. One infant was managed with this strategy for three weeks, by which time sufficient recovery had occurred to permit withdrawal of prostaglandin E1. The other two infants developed right ventricular failure shortly after admission and required extracorporeal membrane oxygenation (ECMO).

Four of the seven infants required ECMO because of failure to respond to escalating doses of inotropic support. Three of these subsequently died. Two died when intensive care was withdrawn after 7-10 days as no ventricular recovery had occurred. (At present, we use ECMO as a bridge to recovery rather than transplantation in the neonatal population because of the shortage of donor organs.) The third death occurred a year after hospital discharge in an infant with poor ventricular function. The single survivor of ECMO has recovered normal ventricular function and is weaning medical treatment.

All three infants who were managed without ECMO survived. One infant has recovered normal ventricular function and is weaning medical treatment. The other two have impaired ventricular function and are receiving medical treatment for cardiac failure. One continues to have a very echo bright appearance in the anterior septum, which probably represents fibrous scarring (fig 2).

Diagnosis of enterovirus infection was by detection of enterovirus RNA by reverse transcriptase polymerase chain reaction of blood $(n=6)$, cerebrospinal fluid $(n=2)$, or throat swabs $(\mathrm{n}=1)$ as previously described. ${ }^{2}$ Maternal blood was tested for enterovirus IgM in three cases and was positive in two cases, suggesting the possibility of both horizontal and vertical transmission. One infant, who subsequently died, was treated with pleconaril, an orally administered antiviral agent with specific activity against the enterovirus family. ${ }^{3}$

\section{DISCUSSION}

Left ventricular dysfunction in the neonatal period is recognised as a complication of sepsis, birth asphyxia, metabolic disease, or structural abnormalities such as aberrant left coronary artery or hypoplastic left heart syndrome. Although myocarditis has been reported as a rare but serious consequence of enterovirus infection in the neonatal period, ${ }^{14}$ the condition is not widely recognised.

Four of the seven cases in this series had global ventricular dysfunction whereas three had severe left ventricular dysfunction, a relatively preserved right ventricle, and duct dependent systemic circulation. The latter group had reduced post-ductal oxygen saturation due to right to left shunting

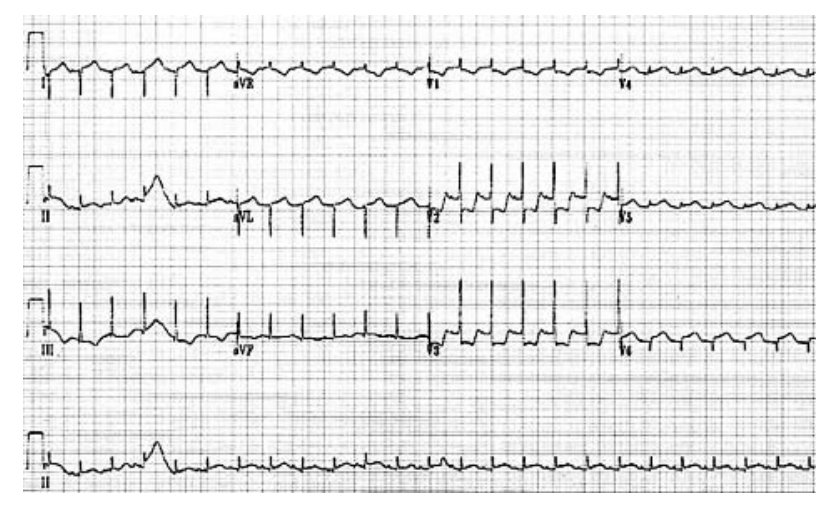

Figure 1 Electrocardiogram showing ST segment depression in II, aVF and the anterior chest leads with poor $\mathrm{R}$ wave progression (case 5). 

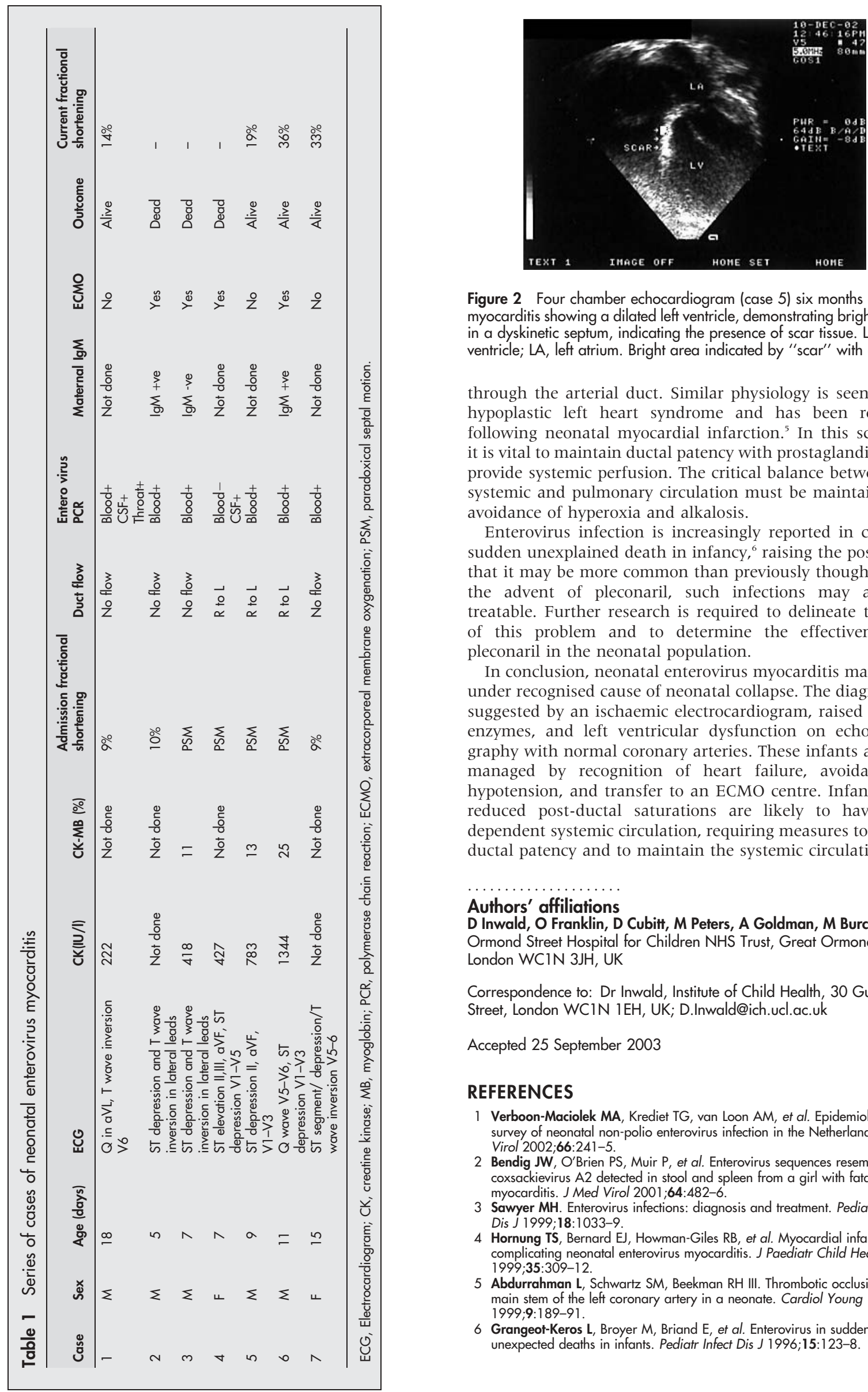

Figure 2 Four chamber echocardiogram (case 5) six months after myocarditis showing a dilated left ventricle, demonstrating bright echoes in a dyskinetic septum, indicating the presence of scar tissue. LV, Left ventricle; LA, left atrium. Bright area indicated by "scar" with arrows.

through the arterial duct. Similar physiology is seen in the hypoplastic left heart syndrome and has been reported following neonatal myocardial infarction. ${ }^{5}$ In this scenario, it is vital to maintain ductal patency with prostaglandin El to provide systemic perfusion. The critical balance between the systemic and pulmonary circulation must be maintained by avoidance of hyperoxia and alkalosis.

Enterovirus infection is increasingly reported in cases of sudden unexplained death in infancy, ${ }^{6}$ raising the possibility that it may be more common than previously thought. With the advent of pleconaril, such infections may also be treatable. Further research is required to delineate the size of this problem and to determine the effectiveness of pleconaril in the neonatal population.

In conclusion, neonatal enterovirus myocarditis may be an under recognised cause of neonatal collapse. The diagnosis is suggested by an ischaemic electrocardiogram, raised cardiac enzymes, and left ventricular dysfunction on echocardiography with normal coronary arteries. These infants are best managed by recognition of heart failure, avoidance of hypotension, and transfer to an ECMO centre. Infants with reduced post-ductal saturations are likely to have duct dependent systemic circulation, requiring measures to ensure ductal patency and to maintain the systemic circulation.

\section{Authors' affiliations}

D Inwald, O Franklin, D Cubitt, M Peters, A Goldman, M Burch, Great Ormond Street Hospital for Children NHS Trust, Great Ormond Street, London WCIN 3JH, UK

Correspondence to: Dr Inwald, Institute of Child Health, 30 Guildford Street, London WCIN 1EH, UK; D.Inwald@ich.ucl.ac.uk

Accepted 25 September 2003

\section{REFERENCES}

1 Verboon-Maciolek MA, Krediet TG, van Loon AM, et al. Epidemiological survey of neonatal non-polio enterovirus infection in the Netherlands. J Med Virol 2002;66:241-5

2 Bendig JW, O'Brien PS, Muir P, et al. Enterovirus sequences resembling coxsackievirus A2 detected in stool and spleen from a girl with fatal myocarditis. J Med Virol 2001;64:482-6.

3 Sawyer MH. Enterovirus infections: diagnosis and treatment. Pediatr Infect Dis J 1999; 18:1033-9.

4 Hornung TS, Bernard EJ, Howman-Giles RB, et al. Myocardial infarction complicating neonatal enterovirus myocarditis. J Paediatr Child Health 1999;35:309-12.

5 Abdurrahman L, Schwartz SM, Beekman RH III. Thrombotic occlusion of the main stem of the left coronary artery in a neonate. Cardiol Young 1999:9:189-91.

6 Grangeot-Keros L, Broyer M, Briand E, et al. Enterovirus in sudden unexpected deaths in infants. Pediatr Infect Dis J 1996;15:123-8. 